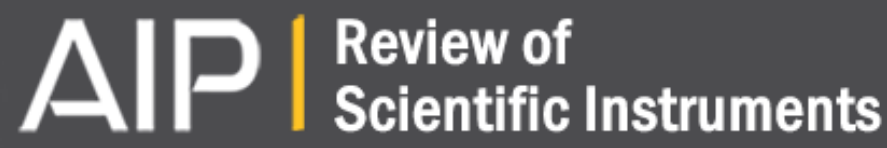

\section{A compact and low-weight sputtering unit for in situ investigations of thin film growth at synchrotron radiation beamlines}

P. Walter, A.-C. Dippel, K. Pflaum, J. Wernecke, J. van den Hurk, J. Blume, and U. Klemradt

Citation: Review of Scientific Instruments 86, 053906 (2015); doi: 10.1063/1.4918620

View online: $\mathrm{http}: / / d x$. doi.org/10.1063/1.4918620

View Table of Contents: http://scitation.aip.org/content/aip/journal/rsi/86/5?ver=pdfcov

Published by the AIP Publishing

\section{Articles you may be interested in}

Influence of plasma-generated negative oxygen ion impingement on magnetron sputtered amorphous $\mathrm{SiO} 2$ thin films during growth at low temperatures

J. Appl. Phys. 111, 054312 (2012); 10.1063/1.3691950

A Two Magnetron Sputter Deposition Chamber Equipped with an Additional Ion Gun for in situ Observation of Thin Film Growth and Surface Modification by Synchrotron Radiation Scattering

AIP Conf. Proc. 879, 1813 (2007); 10.1063/1.2436422

The influence of the growth rate on the preferred orientation of magnetron-sputtered Ti-Al-N thin films studied by in situ x-ray diffraction

J. Appl. Phys. 98, 044901 (2005); 10.1063/1.1999829

Reactive magnetron sputtering of molybdenum sulfide thin films: In situ synchrotron x-ray diffraction and transmission electron microscopy study

J. Appl. Phys. 95, 7665 (2004); 10.1063/1.1736323

A two magnetron sputter deposition chamber for in situ observation of thin film growth by synchrotron radiation scattering

Rev. Sci. Instrum. 72, 3344 (2001); 10.1063/1.1384432

Frustrated by

old technology?

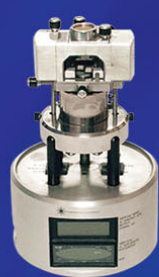

Is your AFM dead and can't be repaired?

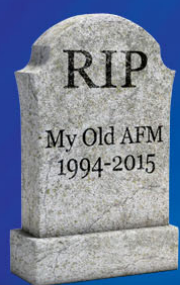

Sick of bad customer support?

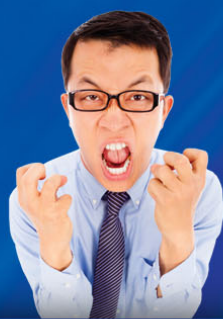

It is time to upgrade your AFM Minimum \$20,000 trade-in discount for purchases before August 31st

Asylum Research is today's technology leader in AFM 


\title{
A compact and low-weight sputtering unit for in situ investigations of thin film growth at synchrotron radiation beamlines
}

\author{
P. Walter, ${ }^{1,2, a)}$ A.-C. Dippel, ${ }^{1,3}$ K. Pflaum, ${ }^{1}$ J. Wernecke, ${ }^{1}$ J. van den Hurk, ${ }^{4}$ J. Blume, ${ }^{1}$ \\ and U. Klemradt ${ }^{2}$ \\ ${ }^{1}$ Deutsches Elektronen-Synchrotron, Notkestr. 85, D-22607 Hamburg, Germany \\ ${ }^{2} 2$ nd. Institute of Physics B and JARA-FIT, RWTH Aachen University, Otto-Blumenthal-Str. 1, \\ D-52074 Aachen, Germany \\ ${ }^{3}$ Center for Materials Crystallography, Department of Chemistry, Aarhus University, \\ Langelandsgade 140, 8000 Aarhus C, Denmark \\ ${ }^{4}$ Institut für Werkstoffe der Elektrotechnik II (IWE II) and JARA-FIT, RWTH Aachen University, \\ Sommerfeldstr. 24, D-52074 Aachen, Germany
}

(Received 16 February 2015; accepted 7 April 2015; published online 13 May 2015)

\begin{abstract}
In this work, we report on a highly variable, compact, and light high-vacuum sputter deposition unit designed for in situ experiments using synchrotron radiation facilities. The chamber can be mounted at various synchrotron beamlines for scattering experiments in grazing incidence geometry. The sample position and the large exit window allow to perform x-ray experiments up to large $q$ values. The sputtering unit is easy to mount on existing experimental setups and can be remote-controlled. In this paper, we describe in detail the design and the performance of the new sputtering chamber and present the installation of the apparatus at different 3rd generation light sources. Furthermore, we describe the different measurement options and present some selected results. The unit has been successfully commissioned and is now available for users at PETRA III at DESY. ( 2015 AIP Publishing LLC. [http://dx.doi.org/10.1063/1.4918620]
\end{abstract}

\section{INTRODUCTION}

For decades, there has been an increasing demand for functional thin films in a large range of applications such as electro-optical devices, ${ }^{1-3}$ solar cell technology ${ }^{4,5}$ or storage devices. ${ }^{6,7}$ According to Moore's Law, ${ }^{8}$ the industrial market for thin films requires the production of more efficient layers with better performance, lower energy consumption, and preferably at lower production cost. Several highthroughput techniques are available to produce thin films ranging from chemical solution deposition $(\mathrm{CSD})^{9,10}$ to vacuum deposition. ${ }^{11,12}$ One of the most intensively used techniques is radio frequency (RF) sputtering ${ }^{13}$ which in comparison to direct current (DC) sputtering ${ }^{14}$ allows to deposit conducting as well as non-conducting materials. The actual film characteristics are largely determined by the deposition parameters such as pressure and gas conditions, power settings, and deposition temperature. ${ }^{15-18}$ However, a general understanding of the underlying physical and crystallographic mechanisms is yet mostly missing. In this context, investigating the film formation in situ during the deposition is particularly useful. Parametric in situ studies of the influence of different processing parameters and different substrate materials, with or without buffer layers, on the thin film evolution are required to develop a general understanding of the underlying physical and crystallographic mechanisms, also in the context of understanding the structure of the interface between the thin film material and the substrate.

Two widely applied in situ techniques to investigate sputter deposited films are photoelectron spectroscopy ${ }^{19,20}$ and

\footnotetext{
a)Electronic mail: peter.walter@desy.de.
}

scanning probe microscopy. ${ }^{21}$ Used in combination, they can deliver an extensive characterisation of the surface beyond its morphology. However, these techniques are limited to only a few nanometres of probing depth and a limitation in time resolution due to energy confinements, low photon flux, or long scanning time. Moreover, usually a large ultra high vacuum (UHV) chamber is necessary to study thin films. By contrast, synchrotron radiation from 3rd generation sources such as PETRA III $^{22}$ offers a broad spectrum of investigation techniques such as spectroscopy, diffraction, or scattering to study surfaces, layers, and interfaces. In addition, the high brilliance of modern synchrotron light sources allows for the observation of the film growth and structure formation at high angular resolution in real time. For this purpose, we developed a highly flexible and compact sputtering chamber to perform grazing incidence diffraction and scattering experiments as well as spectroscopy. These techniques can be conducted simultaneously or subsequently depending on the beamline and chamber setup. In situ sputtering chambers have been built by other groups but they are dedicated for the use at one particular beamline or are specialised for one investigation technique. ${ }^{23-26}$ To the best of our knowledge, no light-weight sputter chambers as presented in this article are reported otherwise. We will report on the operation and performance of our chamber and show some selected results.

\section{REQUIREMENTS}

Most synchrotron radiation beamlines have fixed installed experiment setups such as diffractometers or goniometers, with certain limitations with respect to weight load. Furthermore, permanently installed detectors and sample environ- 
ments lead to a restriction in available space. Consequently, an in situ sputtering chamber has to be minimised in weight and size without limiting the performance of the sputtering process. Generally, in order to allow for sputtering experiments, UHV conditions, e.g., a pressure of $10^{-8} \mathrm{hPa}$ is mandatory to avoid cross contamination or oxidation of the sample or sputtering target but on the other hand, pressures up to $10^{-1} \mathrm{hPa}$ for high pressure sputtering have to be feasible. For many applications, thin films have to be heat-treated in order to induce crystallisation. Therefore, the sputtering unit has to be equipped with a heating stage which can provide temperatures up to $1300 \mathrm{~K}$. In order to perform grazing incidence diffraction and scattering experiments, the exit window for the scattered $\mathrm{x}$ rays has to exhibit a large opening to maximise the observable range in reciprocal space. To realise scattering experiments, the sample has to be mounted accurately in the rotation centre of the setup. Furthermore, the lateral offset between the x-ray beam and diffractometer has to be bridged by an adjustable chamber holder. Considering the limitation of beam time at 3rd generation synchrotron sources, it should be possible to easily and quickly install and remove the chamber. Consequently, the chamber should work as a stand-alone offline unit to offer the possibility to mount the chamber in advance, days before the start of the beam time to do all the necessary cabling, assembling, and tests. A fast transfer to the beamline is mandatory.

In order to combine the powerful techniques provided by high-brilliance synchrotron light sources with standard laboratory techniques such as Auger electron spectroscopy (AES) or low energy electron diffraction (LEED) and reflection highenergy electron diffraction (RHEED), the chamber needs to be integrable on existing UHV analysis chambers such as the MBE chamber of $\mathrm{P}^{2} 8^{27}$ at PETRA III (see Figure 1).

\section{TECHNICAL DESIGN}

\section{A. The sputtering chamber}

The main chamber (see Figure 2) of the modular system can be separated into two parts, the large volume upper part and the bottom part with the support for the sample holder. Both parts are made out of aluminium with custom bimetallic flanges manufactured by Atlas Technologies (Atlas Technologies, 305 Glen Cove Road, Port Townsend, WA 98368). These flanges are made out of a stainless steel sealing face that is explosion-welded (EXW) to an aluminium body. The weight saving of an aluminium chamber in contrast to a chamber fully made out of stainless steel amounts to $7 \mathrm{~kg}$ in

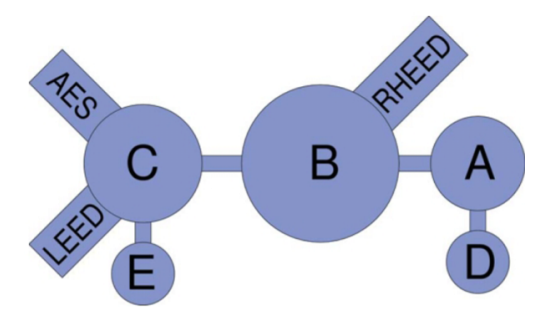

FIG. 1. Sketch of the UHV chamber system in the P08 preparation laboratory with the following chambers: storage and degassing (A), preparation (B) and analysis chamber (C), load lock (D), and the sputtering chamber $(\mathrm{E}){ }^{27}$

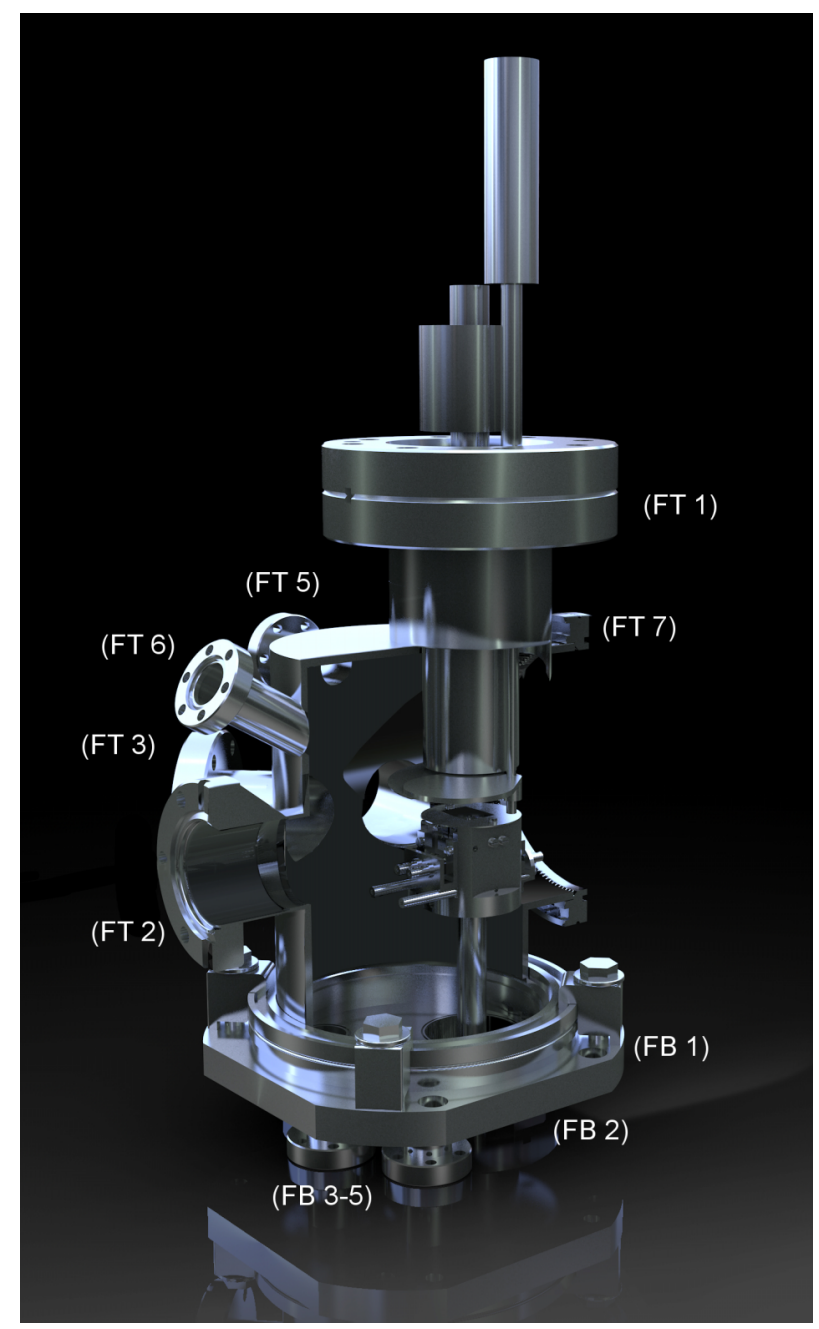

FIG. 2. The sputtering chamber with sputter gun and sample holder. The flanges on top are marked as FT and the flanges at the bottom marked as FB. Labelled as (FT 1) is the flange for the sputter gun. (FT 2) is the flange for the incident X-ray beam. Located with an offset of $45^{\circ}$ to (FT 2) is the flange (FT 3 ), at the far side is the flange (FT 4). Two CF 16 flanges are labelled with (FT 5) and (FT 6). A customised blind flange for the bottom part is marked as (FB 1) with the flange for the sample holder (FB 2) and three concentrically arranged CF 16 flanges (FB 3-5).

this case. Bake-out procedures are realised at temperatures up to $393 \mathrm{~K}$ over 2 days without vacuum leaks due to thermal expansion, which can be a problem especially when bolt connections are realised with different materials. The upper part of the chamber has a diameter of $100 \mathrm{~mm}$ and a height of $155 \mathrm{~mm}$. Seven flanges can be used as infrastructure connectors. One CF 63 flange (FT1) hosts the sputtering gun. If the chamber is used as an advanced vacuum heating stage without the sputtering capability, the corresponding CF 63 flange for the gun can be used for a fluorescence detector in $90^{\circ}$ orientation to the sample. The chamber features, moreover, three CF 40 flanges in different horizontal orientations, all on the sample level, two CF 16 flanges and one DN 100 ISO-K exit window. For the sputtering gun, the CF 63 flange is mounted vertically and $28.5 \mathrm{~mm}$ off-centre towards the exit window. The window for the incident $\mathrm{x}$-ray beam is a CF 40 flange (FT2). CF flanges, in comparison to KF or ISO-K flanges, offer some benefits such as stiffness and stability to 
hold the weight of the whole chamber if needed, e.g., for installation at existing infrastructures, such as the P08 MBE chamber. In addition, CF flanges offer the flexibility to insert different window materials, such as diamond, Kapton ${ }^{\circledR}$ or beryllium. Another advantage to use a flange is the possibility to mount the chamber by a bellow to the evacuated flight tube of the beamline to reduce air scattering. This can be done directly or separated by one of the mentioned window materials. The main chamber is equipped with two more $\mathrm{CF}$ 40 flanges at the same height as the incidence window and the sample. One flange (FT3) has a $45^{\circ}$ offset to the flange of the incoming beam, which allows to mount a bellow that exerts minimum shear force when rotating the chamber or varying the incidence angle $\omega$ on the sample. This flange is usually used to decouple the weight load of the pumping unit (see Sec. III C) from the chamber via a bellow. The third CF 40 flange (FT4) on this level is orthogonal to the incidence window and in line with the sample. Therefore, this flange is in the rotation axis and can be used in different ways, such as sample changing, infrastructure for feed-through, or to mount the chamber to other analysis chambers. Two CF 16 flanges in different orientations are mounted on the chamber. One (FT5) is regularly used as thermocouple feed-through and for pressure measurement and the other one (FT6) is oriented at an angle of $30^{\circ}$ to the beam direction. Therefore, this flange can be used in three ways (i) for spectroscopic measurement equipment (see Sec. VII), (ii) for electrical feed-through to apply an electrical field for determination of, e.g., the dielectric and ferroelectric properties, and (iii) for a gas needle as second gas inlet. The primary gas inlet is located at the gun close to the target (see Sec. III E). This can cause detrimental effects when the primary gas inlet is used with different gases, such as changes in the plasma due to different gas mixtures and to metallisation ${ }^{28}$ of the target when oxygen is used. A second gas inlet is necessary when oxidising conditions are needed, e.g., to induce crystallisation during heating. With a flange in this orientation, the gas needle can be close to the sample without affecting the sputtering process or the x-ray measurement. An ISO-K 100 flange (FT7) is implemented as the exit window for the x-ray beam. The exit window provides the possibility to be equipped with different window materials. Due to the asymmetric arrangement of the sample holder and gun in horizontal direction, the window is located only $40 \mathrm{~mm}$ to the centre of the sample. This design provides an opening angle of $50^{\circ}$ for the diffracted beam. These large accessible diffraction angles are especially of interest for pair distribution function (PDF) ${ }^{29}$ measurements or diffraction experiments using low energy x-rays. In addition, a flight tube can be easily connected to the chamber via the ISO-K flange.

The bottom part is designed for easy installation of the sample holder with respect to electrical feed-through, mounting, and alignment. The top and bottom parts are connected by DN 100 ISO-K flange (FB 1). In fact, the bottom part is a customised blind flange which is directly connected to the sample holder to achieve high precision related to diffractometer movement. In the vertical axis of the sputter gun, there is a CF 40 flange (FB 2) to mount the sample holder which includes the heating stage (Sec. III B). Usually, the sample holder is mounted to a blind flange or a rotation feedthrough with a stepper motor to enable sample rotation, which facilitates sample changing when the chamber is mounted to other analysis chambers or a sample magazine (future upgrade). As support, there are three concentrically arranged CF 16 flanges (FB 3-5). By these three CF 16 flanges, the sample holder including the heating stage is entirely separated from the upper part of the chamber. This fact simplifies the handling during assembling and operation of the sputtering unit.

As a highly flexible apparatus, the weight of the sputtering chamber depends on its particular application and therefore on the installed equipment. The lowest reasonable weight is $12 \mathrm{~kg}$, i.e., for running the unit without sample heating or rotation and no additional feed-through or other equipment. When fully functional, the sputter unit has a weight of $22 \mathrm{~kg}$. This is a tolerable weight for most diffractometers. For diffractometers with a lower weight load, a linkage with a counter weight or a spring as support can be optionally installed. Those options have been used at P08 at PETRA III and MS POWDER at SLS without any negative influence on positioning and rotating.

\section{B. The sample holder and heating stage}

The sputtering unit can be equipped with four different sample holder types. The first, lightest, and simplest solution is an aluminium stack with clamps to fix the sample. With this sample holder, the sample to sputtering target distance can be varied by $\pm 15 \mathrm{~mm}$ but heating is not possible.

The second solution is a UHV Boralectric ${ }^{\circledR}$ heating plate. The maximum substrate size that can be used with this heating stage is $\varnothing 25,4 \mathrm{~mm}\left(1^{\prime \prime}\right)$. Boralectric heating plates (tectra GmbH, D-60323 Frankfurt, Reuterweg 65, Germany) can reach $1773 \mathrm{~K}$ steady state conditions in vacuum and $1273 \mathrm{~K}$ in atmosphere. ${ }^{30}$ In combination with the PID controller (type tectra HC3500) and a low-voltage transformer for small heaters, the heating plate works under stable conditions starting from room temperature (RT) up to maximum temperature. With this heating stage, one can reach heating rates up to $20 \mathrm{~K} / \mathrm{s}$. (All temperature values per second are the mean values by ramping from RT up to $1073 \mathrm{~K}$ or vice versa.)

An electron beam (ebeam) heating device can be installed as the third solution. Using the ebeam heating, the maximum wafer size is $13 \times 10 \mathrm{~mm}^{2}$. A pressure lower than $5 \times 10^{-5} \mathrm{hPa}$ is required to avoid flash-overs. Therefore, the ebeam heater cannot be operated at elevated temperatures during sputtering or post-annealing in oxygen, argon, or any other plasma gas or mixed atmospheres. The advantage of an ebeam heating is the maximum temperature of more than $1700 \mathrm{~K}$ and heating rates up to $50 \mathrm{~K} / \mathrm{s}$.

As the forth sample holder for annealing purposes, a passively (non-cooled) or actively cooled version of a halogen lamp heating device is available (see Figure 3). Heating with a $150 \mathrm{~W}$ halogen lamp, one can reach a substrate temperature of $1173 \mathrm{~K}$ and heating rates up to $3 \mathrm{~K} / \mathrm{s}$. In contrast to the ebeam heating device, this heating solution is completely independent of the chamber pressure. The actively cooled version of this heating stage can achieve cooling rates up to 


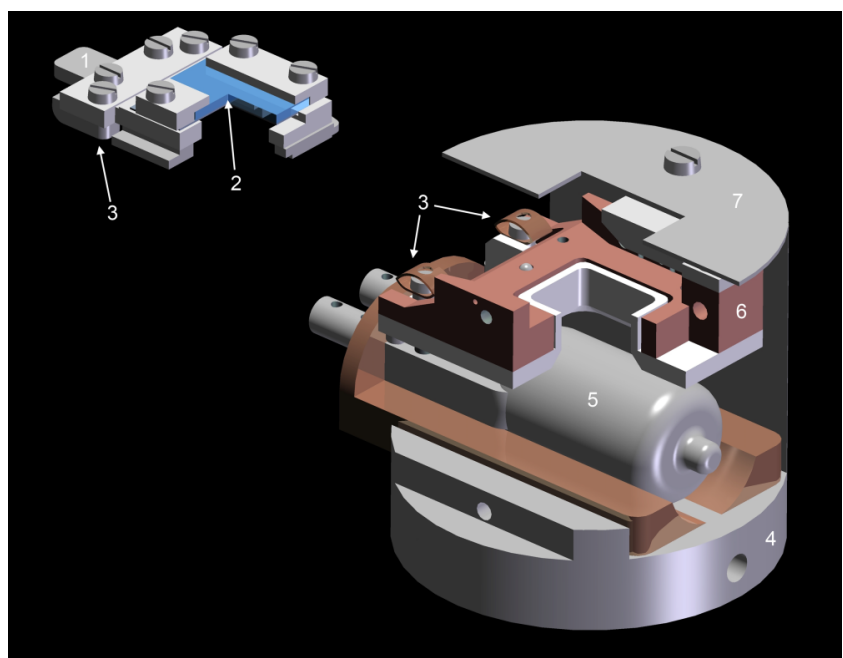

FIG. 3. View of the sample holder and passively cooled heating stage with halogen lamp. Marked (1) is the sample changer to enable the connection to the P08 MBE chamber and the future upgrade of a sample magazine. The substrate is marked as (2). To realise temperature measurement and sample changing, the sample holder is equipped with sliding contacts (3) for the thermocouple. (4) is the heating stage holder with halogen lamp (5), sample cradle (6), and an optional heating shield (7).

$8 \mathrm{~K} / \mathrm{s}$ and a minimum temperature of $253 \mathrm{~K}$ can be reached by circulating cold nitrogen gas through the cooling lines. By varying the stack length of the sample holder for the heating devices, the substrate-to-target distance can be changed to $\pm 10 \mathrm{~mm}$.

For a larger substrate-to-target distance, a spacer can be implemented at flange (FT7). By doing this, a maximal distance of $80 \mathrm{~mm}$ can be realised this way.

\section{The vacuum system}

The vacuum system has two main parts, the controllable gas inlet and the turbo pump station with a pressure sensor controlled valve. To regulate the gas flow, a commercial gas flow controller (type MF1) which is configured for argon (Ar) by the company MKS Instruments (MKS Instruments Deutschland GmbH, Schatzbogen 43, 81829 München, Germany) is used. This gas flow controller is specially configured for monatomic gases like Ar and has an accuracy of $\pm 0.5 \%$ with respect to the displayed value. In combination with a controller (type MKS PR4000B), the gas flow can be adjusted from 0.5 standard cubic centimetres per minute (SCCM) to 20 SCCM. The gas inlet is a fixed part of the sputter gun. Therefore, the gas stream ends right beneath the target. This means that the highest gas concentration is located close to the plasma. Due to three concentrically arranged gas inlets, the gas afflux is homogeneous. As a result, an optimised plasma process and hence a homogeneous deposition process are facilitated. The aspiration is managed by a Pfeiffer (Pfeiffer Vacuum GmbH, Berliner Strasse 43, 35614 Asslar, Germany) pumping station (type High Cube Eco 80). To establish stable pressure conditions, a butterfly valve (type MKS 253B) is installed in front of the pumping station in accordance with a Baratron ${ }^{\circledR}$ (type MKS 627D) to measure the pressure in the chamber. When combining both options, a pressure stability of $1 \%$ with respect to the displayed value is obtained.
As mentioned in Sec. III A, in order to avoid contamination of the gas line when different gases are used in the same line or metallisation of the target when oxygen is used, a second gas inlet can be installed (FT6). The second gas inlet may be equipped with a needle valve or a gas flow controller (type MF1) which is configured for oxygen $\left(\mathrm{O}_{2}\right)$. With a second installed gas inlet, one can have the gas outlet close to the sample.

\section{The controlling system}

The whole sputtering system can be controlled with a self developed TAURUS control program. TAURUS is a framework for Tango 3D command-line interface (CLIs) and 3D graphical user interface (GUIs), based on Python and PyQT. All devices such as the sputter gun controller, the matchbox, or the MKS vacuum controller are handshake-controlled via Tango servers. Tango ${ }^{31}$ is an object oriented distributed control system using CORBA, it is developed as a collaborative effort between ALBA, ANKA, DESY, ELETTRA, ESRF, FRM II, MAX-IV, SOLARIS, and SOLEIL institutes (http://www.tango-controls.org/). All controller units are installed in a single $19^{\prime \prime}$ electronics rack. This simplifies the transport and the setup procedures at the beamline or offline at a laboratory. This rack is placed close to the chamber; therefore, no cables have to go through a chicane. The rack can be remotely controlled from the control hutch via local network access or direct cable connection and it works independently from the operating system installed on the host computers. Alternatively, all devices can be operated manually at the rack. The GUI controls the sputtering process, the heating stage, and the vacuum system. Beamline controls such as for stepper motors are not part of the installation in order to keep the flexibility and implementation possibilities at different beamlines at different synchrotron sources.

\section{E. The sputtering system}

The sputtering chamber can be equipped with conventional CF 40 or CF 60 sputter sources. We use a magnetron sputtering source (Type ST 10) from AJA (AJA International, Inc., 809 Country Way, North Scituate, MA 02060, USA). The sputter gun is water cooled and can work with a power from $1 \mathrm{~W}$ to $300 \mathrm{~W}$ in $\mathrm{DC}$ and RF mode. The target size is limited to $1^{\prime \prime}$. The sputter gun provides a compressed air guided shutter. Table I denotes the main parameters of the sputtering chamber.

\section{EXPERIMENTAL METHODS}

With the above described sputtering chamber, it is possible to study the sputter deposition process from the first interface formation and follow it to the final stage. Therefore, different in situ X-ray techniques can be performed with this chamber.

- In situ grazing incidence small angle $\mathrm{x}$-ray scattering (GISAXS) experiments allow for studies of the sputter deposition process already from the early stages of 
TABLE I. This table illustrates the main parameter of the sputtering unit.

\begin{tabular}{lc}
\hline \hline Parameter & Value \\
\hline Target size (in.) & 1 \\
Sputtering power (W) & $1-300$ \\
Sputtering gas & $\mathrm{Ar},\left(\mathrm{Ar}, \mathrm{O}_{2}\right)$ mixture, ${ }^{\mathrm{a}} \mathrm{N}_{2}$ \\
Base pressure $(\mathrm{hPa})^{\mathrm{b}}$ & $1 \times 10^{-6}$ to $5 \times 10^{-8}$ \\
Sputtering pressure $(\mathrm{hPa})$ & $1 \times 10^{1}$ to $1 \times 10^{-3}$ \\
Target— - substrate distance (mm) & $40 \pm 10^{\mathrm{c}}$ \\
Gas flow (SCCM) & $0.5-22$ \\
Annealing temperature $(\mathrm{K})$ & $\mathrm{RT}-1700$ \\
\hline \hline
\end{tabular}

${ }^{\text {a }}$ Mixture is continuously adjustable due to two MKS flow controllers.

${ }^{\mathrm{b}}$ Depending on the window material.

${ }^{\mathrm{c}}$ Depending on the sample holder.

deposition. ${ }^{32,33}$ If heat-treatment is needed to initiate crystallisation, one can perform GISAXS before, during, and after the crystallisation. Therefore, GISAXS gives a deep understanding of the influence of process parameters on the grain size, shape, size distribution, and correlation lengths.

- In situ X-ray powder diffraction (XRPD) provides information about phases or phase transitions, crystallinity, and lattice parameters and can be used for crystal structure solution and refinement. In addition, in situ XRPD is the basis to derive the crystallisation pathway and transformation kinetics during annealing of the deposited layers. In contrast to GISAXS, this method is sensitive to atomic distances.

- In situ X-ray reflectivity (XRR) measurements provide the film thickness, surface and interface roughness, and electron density as well as their changes due to the crystallisation process.

These techniques provide the possibility to study many aspects of thin films in situ and to analyse the dependency of the film development on the processing parameters.

Furthermore, x-ray absorption spectroscopy measurements are possible in backscattering direction with the CF 16 flange (FT6) in perpendicular direction with the CF 63 flange (FT1) or under grazing incidence through the exit window (FT7). Experiments with these techniques are foreseen (see Sec. VII).

In addition, when the chamber is not used with a synchrotron beam, the unit can be attached to the MBE chamber of P08 (see Fig. 1). In this way, surface investigation by LEED, RHEED, and AES can be performed.

\section{INSTALLATION OF THE SPUTTERING UNIT AT DIFFERENT SYNCHROTRON BEAMLINES}

We have proven that this sputtering unit is capable to be used at different synchrotron beamlines. Our sputtering system is mainly designed to be installed at the P02.1 high resolution powder diffraction beamline ${ }^{34}$ at the PETRA III storage ring. In addition, it also works at various beamlines at different synchrotron facilities. Aside from synchrotron beamlines and the MBE chamber of P08 (see Sec. IV), the chamber can be used at any laboratory where sufficient free space is available.
The installation at beamlines where the sputtering unit has successfully been used is described in this section.

\section{A. Beamline P02 at PETRA III, DESY}

The chamber was originally designed for the beamline P02.1 at PETRA III for high resolution and high photon energy powder diffraction. ${ }^{34}$ This beamline combines the advantages of high resolution and high energy provided by one of the most brilliant synchrotron light sources, PETRA III. These capabilities make P02.1 a powerful tool for in situ timeresolved measurements (see, e.g., Refs. 35 and 36). P02.1 has a fast working (15 frames/s) large area 2D detector (Model XRD 1621, PerkinElmer, Waltham, MA, USA) mounted on a precise motorised table. With this detector table, sample to detector distances from $200 \mathrm{~mm}$ up to $3000 \mathrm{~mm}$ are available for use. P02.1 runs at a fixed photon energy of $60 \mathrm{keV}$ and currently without focusing optics (future upgrade). For grazing incidence measurements using the sputtering unit, the detector is positioned in a way that the beam hits the middle of the lower edge of the active detector area and at a distance that the full angular range of the powder pattern, which is limited by the exit window, is observable. This leads to a maximum utilisation of the active detector area. The chamber is mounted on the $\omega$ circle of the high resolution diffractometer, which is the inner of three concentric circles (see Figure 4). The chamber can be tilted in $\omega$ with an accuracy on the order of $10^{-3}$ degrees. Based on the high resolution, which is of special interest for Rietveld refinement, and the high energy of P02.1, data can be collected up to high $q$ values in reciprocal space, which are especially needed for PDF. However, with this setup, the upper $q$ limit in a single powder pattern is $25 \AA^{-1}$ by using the $2 \mathrm{D}$ detector. The maximum load capacity for the diffractometer $\omega$ plate is $50 \mathrm{~kg}$, which is reduced by half when using the pre-installed xyz-stage. As mentioned above, the remaining capacity of $25 \mathrm{~kg}$ is sufficient to implement the chamber with all heating and sample manipulation options.

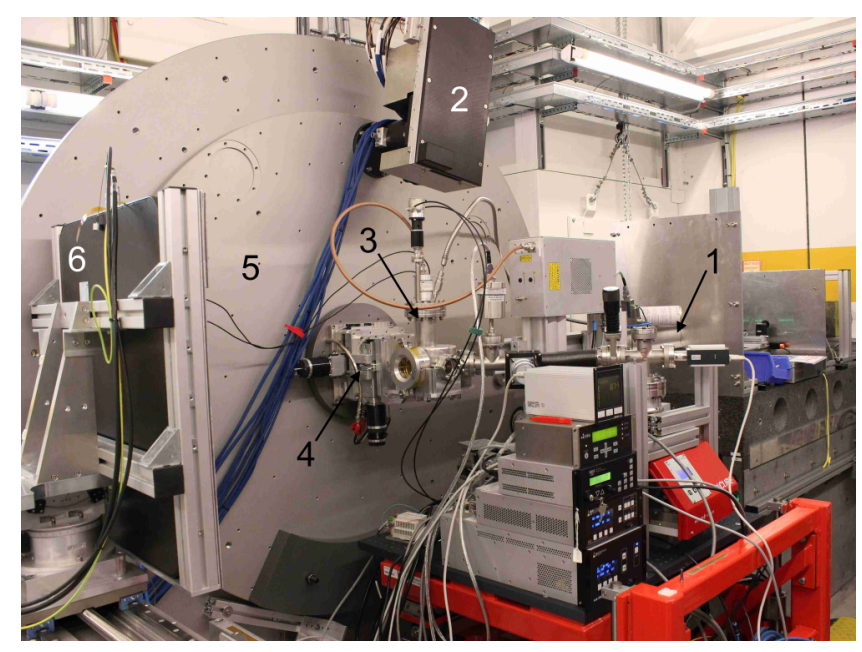

FIG. 4. The sputtering chamber installed at the high resolution powder diffraction beamline P02.1. The photograph shows: (1) X-ray flight tube, (2) multi-crystal analyser detector, (3) sputtering unit, (4) $\omega$ circle and xyz-stage, (5) high resolution diffractometer and (6) large area detector. 


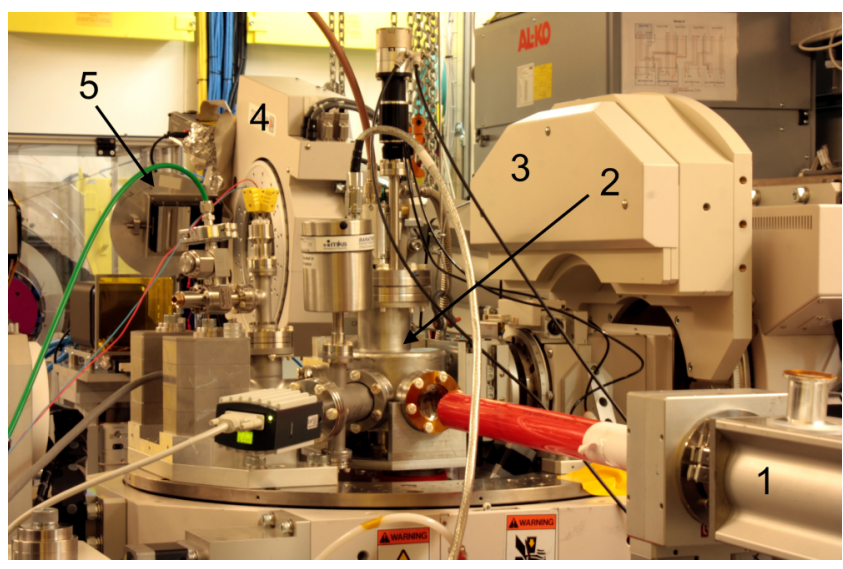

FIG. 5. The sputtering chamber installed at the high-resolution diffraction beamline P08. The photograph shows: (1) x-ray flight tube, (2) sputtering unit, (3) six-circle diffractometer, (4) high precision detector arm, and (5) strip detector.

\section{B. Beamline P08 at PETRA III, DESY}

The second beamline, where we used our sputtering system, is the high-resolution diffraction beamline P08. ${ }^{27}$ In contrast to the P02.1 beamline, $\mathrm{P} 08$ has a tunable $\mathrm{x}$ ray energy (5.4-29.4 keV), focusing optics, and a six-circle diffractometer. This diffractometer is specifically designed to accommodate the beam level at $900 \mathrm{~mm}$ above the floor and offers extremely high precision. The maximum weight load of this diffractometer at the sample position is $10 \mathrm{~kg}$. Therefore, we used a balance weight guided through two deflection pulleys. With this simple solution, we reduced the weight load to less than $10 \mathrm{~kg}$ without limitation on the chamber and its performance and without inducing shear forces on the diffractometer (see Figure 5). Part of the detector standard equipment is a $1280 \times 50 \mu \mathrm{m}^{2}$ strip detector (Model Mythen $1 \mathrm{k}$, Dectris, Baden, Switzerland), a single-photon counting pixel detector with $487 \times 619$ pixels of $172 \mu \mathrm{m}^{2}$ each (Model Pilatus 300k, Dectris, Baden, Switzerland), and a hybrid photon counting detector with $1030 \times 1065$ pixels of $75 \mu \mathrm{m}^{2}$ each (Model Eiger 1M, Dectris, Baden, Switzerland). All of these detectors were used at different beam times with the sputtering unit. These detectors can either be used mounted on the movable detector arm of the diffractometer in a sampleto-detector distance of $1050 \mathrm{~mm}$ or fixed mounted on a table with $2 \mathrm{~m}$ distance. With the line detector, it is easy to perform XRR and XRPD measurements either in time-resolved mode with limited $2 \theta$ range or over the whole possible $2 \theta$ range by scanning with the detector arm of the diffractometer. With the area detectors (Pilatus/Eiger), one can perform XRPD, $\mathrm{XRR}$, and GISAXS measurements in situ without any detector movement. The simultaneous use of an area detector with the stripe detector is also possible. This combination allows to do time-resolved in situ GISAXS with the area detector and time-resolved in situ XRPD at a selected $2 \theta$ range above the GISAXS regime.

\section{MS-POWDER at SLS}

To use the advantages of a stripe detector over a wide $2 \theta$ range, the chamber was installed at the MS-POWDER

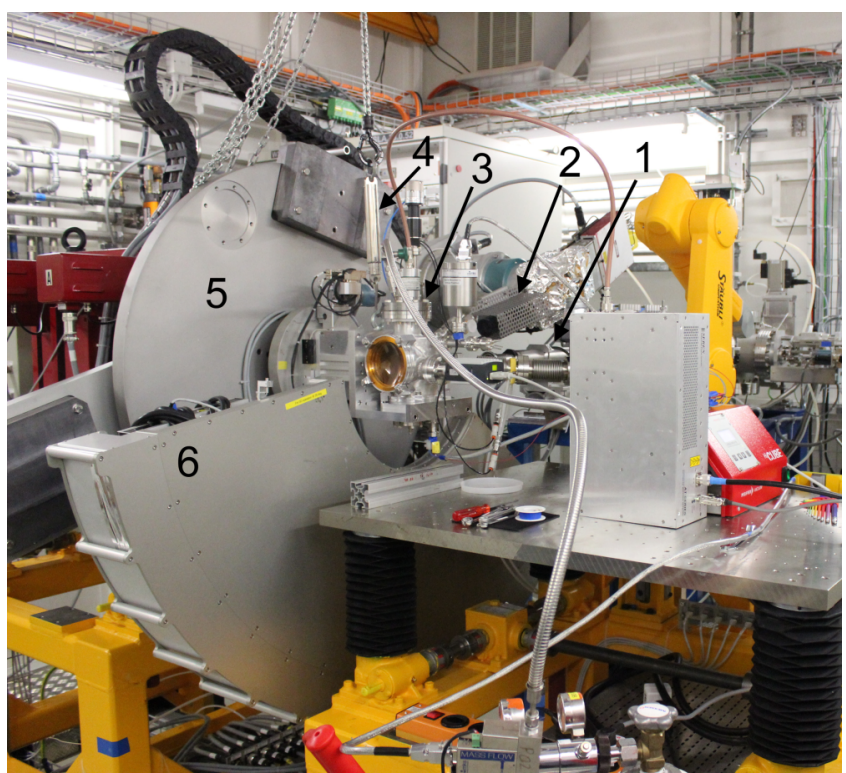

FIG. 6. The sputtering chamber installed at the MS-POWDER beamline at SLS. The photograph shows: (1) x-ray flight tube, (2) VGA camera, (3) sputtering unit, (4) spring balance connected to the experimental hutch crane, (5) high resolution diffractometer, and (6) $120^{\circ}$ strip detector.

beamline ${ }^{37}$ at the Swiss Light Source (SLS) (see Figure 6). The line detector (Model Mythen II, Dectris, Baden, Switzerland) consists of 24 modules and covers an angle of $120^{\circ}$. In comparison with a commercial Mythen type detector, as the one at P08, one is able to do time resolved in situ XRPD measurements over the whole $2 \theta$ range of interest without moving the detector. The MS-POWDER beamline offers tunable energy (5-40 keV), focusing optics, and a two-circle high resolution diffractometer. The distance from the x-ray beam to the $\omega$ plate is $275 \mathrm{~mm}$. To mount the sputtering chamber at the sample plate, we used two aluminium profiles and a spring balance connected to the experimental hutch crane to reduce the weight load on the diffractometer. In this way, we were able to use the precise $\omega$ movement of the diffractometer without inducing shear forces due to weight overload.

\section{PDIFF at ANKA}

Another instrument, where we successfully tested the flexibility of our sputtering system, is the powder diffraction (PDIFF) beamline at ANKA-the synchrotron radiation facility at the Karlsruhe Institute for Technology (KIT) (see Figure 7). This beamline delivers a focused monochromatic x-ray beam with selectable energy $(6-21 \mathrm{keV})$ and a heavy duty 3-circle powder diffractometer capable of carrying sample loads up to approximately $60 \mathrm{~kg}$ together with multiple detector systems. During our beam time, we used the CCD detector (Model Princeton X-ray camera, Princeton Instruments, New Jersey, USA). Due to the heavy duty diffractometer, no weight compensation was necessary.

\section{SELECTED RESULTS}

Our first scientific experiments focused on barium titanate $\left(\mathrm{BaTiO}_{3}\right) . \mathrm{BaTiO}_{3}$ may be considered the "classic" perovskite 


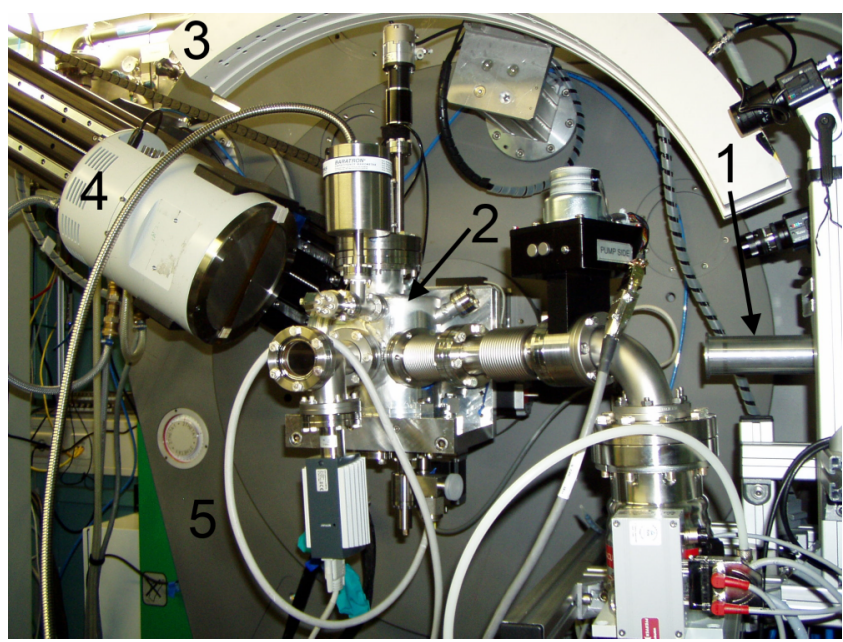

FIG. 7. The sputtering chamber installed at the high-resolution diffraction beamline PDIFF at ANKA. The photograph shows: (1) x-ray flight tube, (2) sputtering unit, (3) $90^{\circ}$ curved PSD detector, (4) Princeton CCD detector, and (5) high resolution diffractometer.

type ferroelectric material from a historical point of view ${ }^{38-40}$ since it was the first material of this type in which the $\mathrm{B}$ site cation displacement was discovered to be the origin of ferroelectricity. ${ }^{41-43}$ It shows ferroelectric behaviour at room temperature and has outstanding properties with respect to ferroelectricity, piezoelectricity, and a high dielectric constant. ${ }^{44,45}$ Pure and chemically modified $\mathrm{BaTiO}_{3}$ powders and thin films have been used as the functional component in various electrical applications for several decades. ${ }^{46}$ Recently, it was found that even single unit cell high $\mathrm{BaTiO}_{3}$ layers were ferroelectric under appropriate boundary conditions, ${ }^{47,48}$ which makes $\mathrm{BaTiO}_{3}$ a promising material for minimising ferroelectric-ferromagnetic storage devices. ${ }^{49}$ The following properties make $\mathrm{BaTiO}_{3}$ an ideal material for initial experiments with an in situ sputtering unit: (i) it is a well known material, which makes it easy to compare the results with the literature and (ii) the need of tailoring more efficient and thinner layers makes $\mathrm{BaTiO}_{3}$ a material of continued interest and ever increasing demands that only can be clarified by in situ experiments. Independent of the needed application, the first step of $\mathrm{a} \mathrm{BaTiO}_{3}$ thin film is the initial interaction with the substrate surface and the subsequent growth of an amorphous layer.

Figure 8 shows a waterfall plot of the scattered x-ray intensity recorded during the amorphous growth of a $\mathrm{BaTiO}_{3}$ film on $\mathrm{a} \mathrm{SiO}_{2}$ wafer by a waterfall plot of XRPD patterns. The wafer was base cleaned, an $\mathrm{Ar} / \mathrm{O}_{2}$ gas mixture of 4:1 was used during sputtering, the gas flow was 1.5 SCCM, and the deposition pressure was $2 \times 10^{-2} \mathrm{hPa}$ with a power of $\mathrm{P}=0.02 \mathrm{~W} / \mathrm{cm}^{2}$. Data were taken at the MS-Powder beamline at the Swiss Light Source at an incidence angle of $\omega_{i}=5^{\circ}$ and a sample-to-detector distance of $1372 \mathrm{~mm}$. By using the $120^{\circ}$ Mythen detector, we recorded frames over $10 \mathrm{~s}$ each. Depicted is the continuous growth of the amorphous $\mathrm{BaTiO}_{3}$ with a deposition rate of $1.5 \mathrm{~nm} / \mathrm{min}$ at a wafer temperature of $333 \mathrm{~K}$. The broad peaks indicate mostly diffuse scattering because it is not concentrated into a few Bragg peaks but equally distributed over a large range of scattering angles $2 \theta$. This characteristic diffraction pattern for an amorphous film is caused by its short range order; the long range order as in a crystalline phase is missing. In an amorphous solid, only the radii of the first few bonding spheres are well defined, whereas with increasing distance from the central atom, both the distance and the symmetric arrangement of atoms on the spheres become washed out. The intensity of the amorphous peak is roughly proportional to the film thickness. We measured the final thickness using XRR at P08 and obtained $98.5 \mathrm{~nm}$.

The crystallisation of $\mathrm{BaTiO}_{3}$ depends on the film thickness, the film-substrate interface behaviour, and the substrate temperature. Figure 9 shows the x-ray signal during the

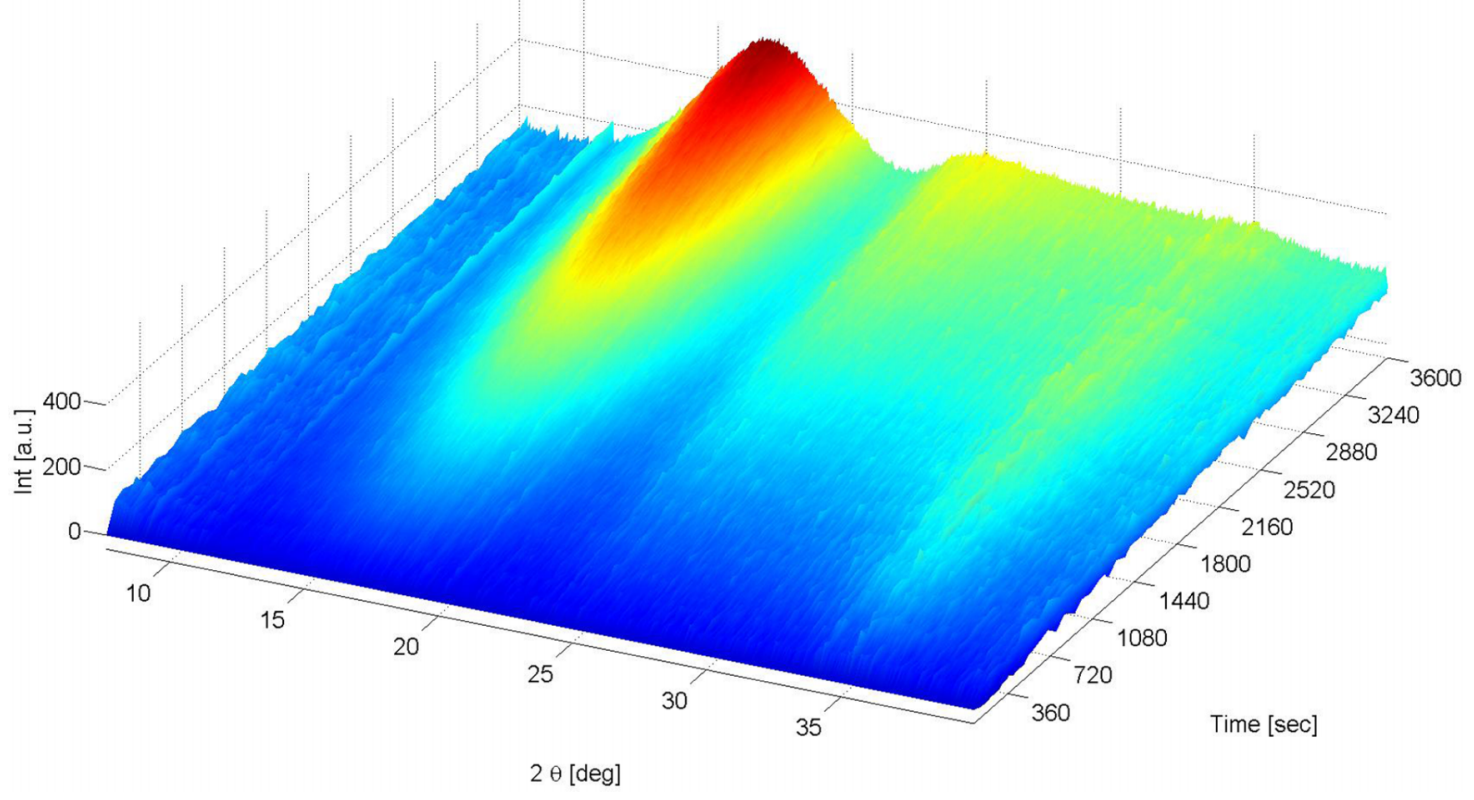

FIG. 8. Time-resolved XRPD patterns collected during the growth of an amorphous $\mathrm{BaTiO}_{3}$ film. It is characterised by only a short range order, the long range order as in a crystallite is missing. Measured at MS-Powder at SLS. 


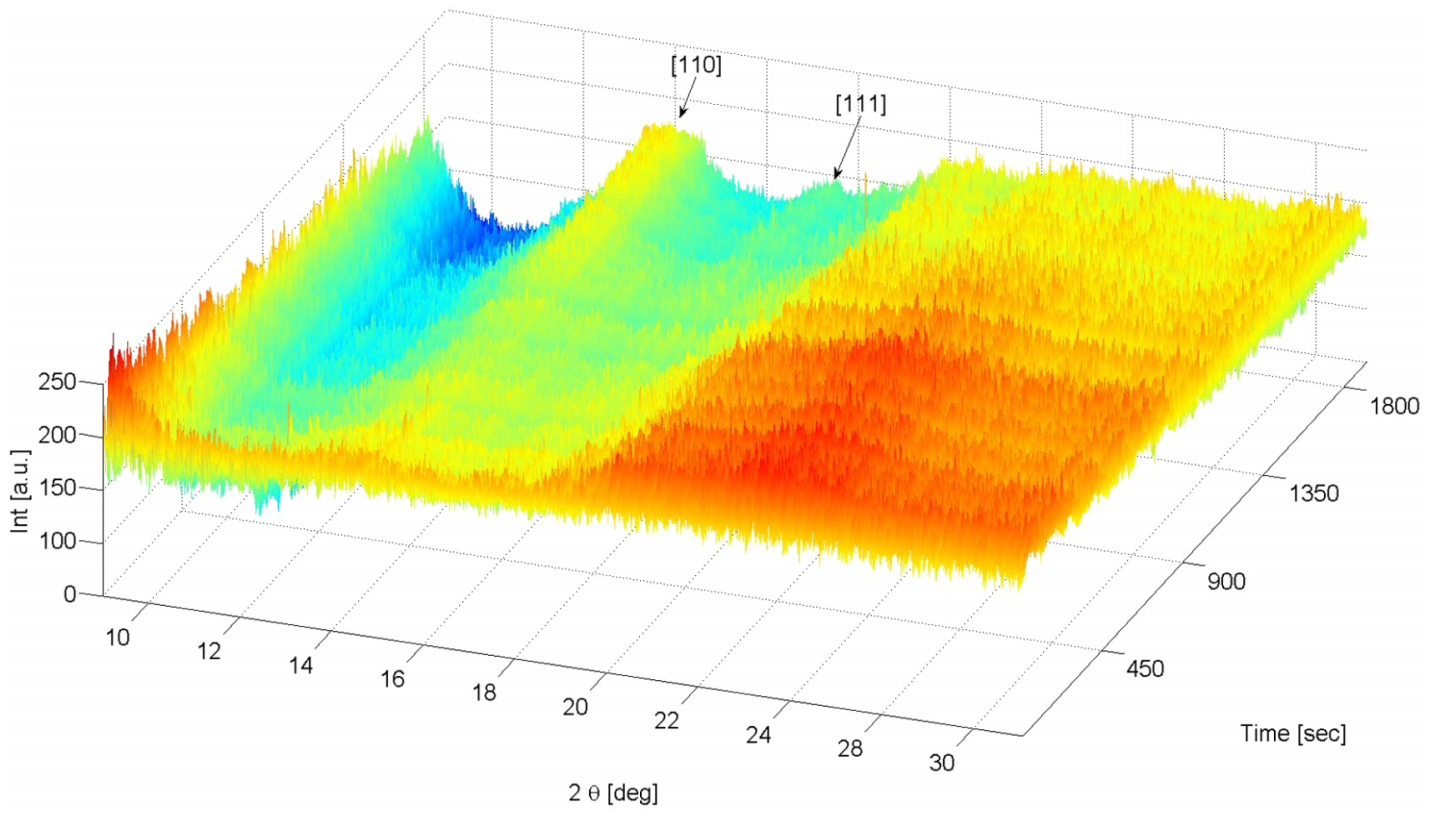

FIG. 9. XRPD patterns collected during sputtering at the same deposition parameters as in Figure 8 but at a different substrate temperature. The deposition took place at $673 \mathrm{~K}$. Measured at PDIFF at ANKA.

growth of a $\mathrm{BaTiO}_{3}$ film at a substrate temperature of $673 \mathrm{~K}$. We used exactly the same sputtering conditions as for the experimental data shown in Figure 8, except for the substrate temperature. The measurements were carried out at the PDIFF beamline at ANKA. By using the CCD-camera, we recorded frames with $10 \mathrm{~s}$ exposure time each. As reported ${ }^{50}$ when annealing a sputtered layer at the crystallisation temperature,

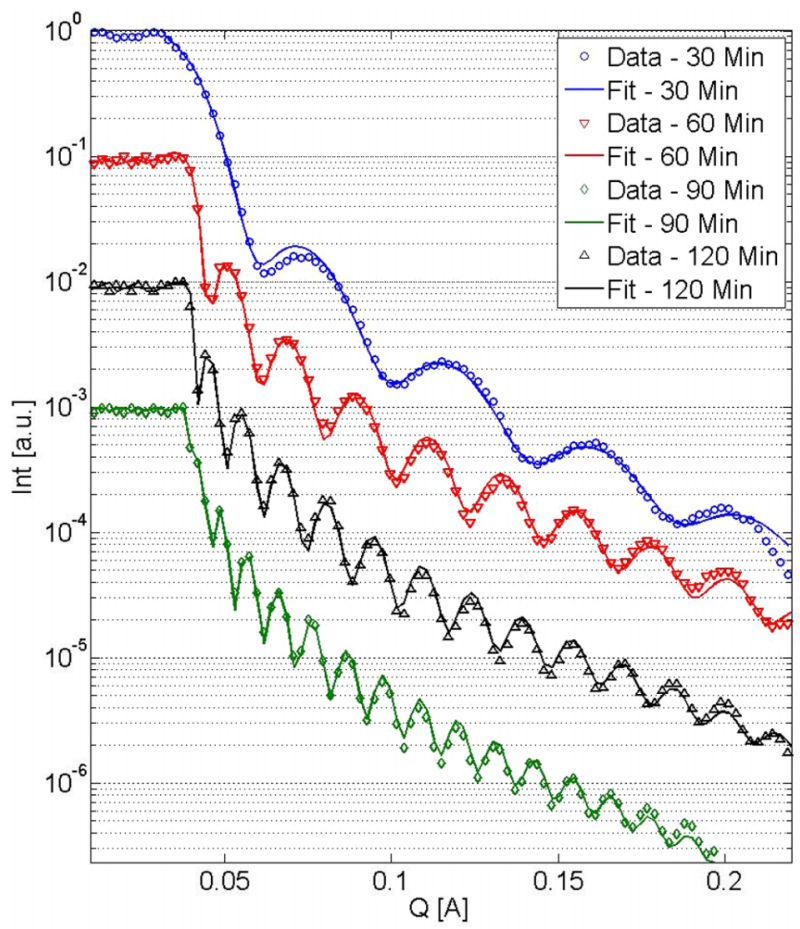

FIG. 10. XRR data collected at P08 at PETRA III. Each measurement was carried out after steps of $30 \mathrm{~min}$ of deposition. The sputtering power was $8 \mathrm{~W}$, the target-to-substrate distance was $40 \mathrm{~mm}$, the substrate temperature was $313 \mathrm{~K}$, and the sputtering pressure was $1 \times 10^{-2} \mathrm{hPa}$. the perovskite phase of $\mathrm{BaTiO}_{3}$ thick films $(>500 \mathrm{~nm})$ started to nucleate in the bulk of the films simultaneously with the nucleation of the intermediate phase at the interface. During annealing of our films, the perovskite structure forms. The broad Bragg peaks suggest the presence of a nano crystalline structure in the annealed thin film.

Figure 10 shows examples of XRR measurements during continuous deposition progress taken at P08 with an X-ray energy of $25 \mathrm{keV}$, a sputtering power of $5 \mathrm{~W}$, and a pressure of $2 \times 10^{-2} \mathrm{hPa}$. Data analysis using Parratt's formalism ${ }^{51}$ shows a constant growth, a slight increase of surface roughness, and mass density with increasing deposition time (see Table II). The higher surface roughness after $30 \mathrm{~min}$ can be explained by incomplete surface coverage. For the simulations, the data were normalised and an illumination correction was done.

To verify the XRR results, we performed cross-sectional scanning electron microscope (SEM) measurements. Figure 11 shows a SEM image of the cross-section of a $33 \mathrm{~nm}$ thick $\mathrm{BaTiO}_{3}$ layer deposited on a $\mathrm{Si}$ wafer with a $450 \mathrm{~nm}$ oxide layer. It shows a $\mathrm{BaTiO}_{3}$ layer thickness of $\sim 33 \mathrm{~nm}$ in in good agreement with the XRR results from P08.

A detailed analysis is under way and not in the scope of this paper.

Table III denotes a matrix of deposition rates of $\mathrm{BaTiO}_{3}$ depending on the pressure and the sputtering power. These two parameters have the largest impact on the deposition rate.

TABLE II. Layer thickness, density, and roughness for $\mathrm{BaTiO}_{3}$ sputtered at $\mathrm{P}=0.02 \mathrm{~W} / \mathrm{cm}^{2}$

\begin{tabular}{lccc}
\hline \hline Time & Layer thickness $(\mathrm{nm})$ & Density $\left(\mathrm{g} / \mathrm{cm}^{3}\right)$ & Roughness \\
\hline $30 \mathrm{~min}$ & 13.83 & 2.96 & 2.21 \\
$60 \mathrm{~min}$ & 29.65 & 3.25 & 1.98 \\
$90 \mathrm{~min}$ & 39.90 & 3.29 & 2.00 \\
$120 \mathrm{~min}$ & 52.48 & 3.31 & 2.28 \\
\hline \hline
\end{tabular}




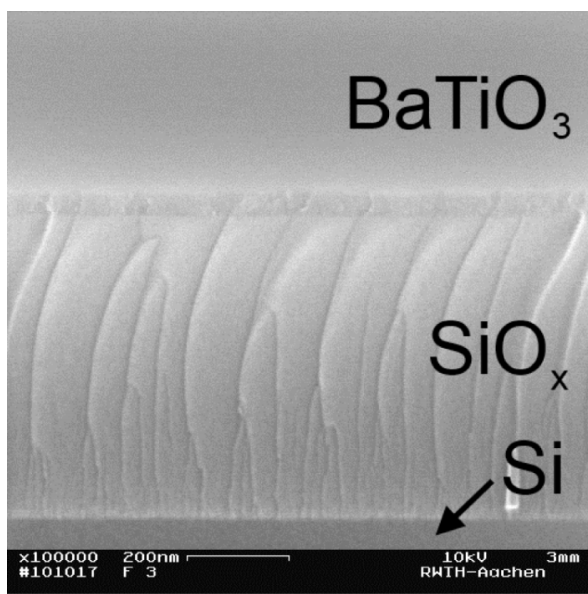

FIG. 11. Scanning electron micrograph of a $33 \mathrm{~nm} \mathrm{BaTiO}_{3}$ film on a Si wafer with a $450 \mathrm{~nm}$ oxide layer. The film thickness was also measured via XRR at P08 and matches with the thickness of $33 \mathrm{~nm}$.

TABLE III. This table illustrates the deposition rate in dependency on the sputtering power for a $1^{\prime \prime}$ target and chamber pressure for $\mathrm{BatiO}_{3}$ in $\mathrm{nm} / \mathrm{min}$ (mean value over 3 measurements).

\begin{tabular}{lccc}
\hline \hline & $1 \times 10^{-3} \mathrm{hPa}$ & $1 \times 10^{-2} \mathrm{hPa}$ & $1 \times 10^{-1} \mathrm{hPa}$ \\
\hline $5 \mathrm{~W}$ & 0.4 & 0.6 & 0.4 \\
$10 \mathrm{~W}$ & 1.2 & 1.4 & 1.3 \\
$15 \mathrm{~W}$ & 1.9 & 2.1 & 2.0 \\
\hline \hline
\end{tabular}

\section{CONCLUSIONS AND OUTLOOK}

To summarise, we reported on the design and installation of a highly flexible and compact sputtering unit which can be used for in situ studies via x-ray scattering and spectroscopy. The position of the sample in the sputtering chamber allows for simultaneous deposition and grazing incidence $\mathrm{x}$-ray scattering. This offers unique possibilities to study in situ interface formation, cluster formation, and thin film growth. The sputtering chamber has been successfully integrated into various beamlines at different synchrotron sources and generates promising results. Different heating stages for varying applications are available. The modularity makes this sputtering unit a versatile tool for in situ investigations of interface formation and thin film growth at different synchrotron beamlines, as well as offline as a stand-alone apparatus or in combination with other laboratory analysis chambers such as the MBE chamber of P08 at PETRA III.

Future measurements to perform $\mathrm{x}$-ray absorption spectroscopy methods during sputtering are planned. In addition, we foresee to investigate the influence of mechanical stress induced by an electric field $\mathrm{E}$ in situ during the deposition process. In this way, the permanent dipoles of the room temperature tetragonal phase of $\mathrm{BaTiO}_{3}$ are aligned while the material is deposited. Hence, possible stress effects that are caused by the initial collective alignment of randomly oriented dipoles during the first polarisation are avoided. In addition, the growth at the interface between substrate and film is likely to differ from the bulk, e.g., with respect to defect density and orientation selection. By structural XRPD as well as electrical characterisation, we will investigate these potentially favourable effects on the ferroelectric performance of the films.

\section{ACKNOWLEDGMENTS}

The authors gratefully acknowledge technical support from L. Wilke, M. Fleck, H. Zink, and the FS-EC group. C. Deiter, D. Reuther, F. Bertram, R. Doehrmann, K. Schlage, and O. Seeck from the FS-PE group are thanked for fruitful discussions and help during the different beam times. For help in creating the graphic Fig. 2, we are indebted to J. Frerichs. Some experiment were performed on the MS-X04SA: Materials Science beamline at the Swiss Light Source, Paul Scherrer Institut, Villigen, Switzerland. Therefore, we are grateful to N. Casati at Swiss Light Source for the efforts during the experiment. We acknowledge the Synchrotron Light Source ANKA for provision of instruments at their beamlines and we would like to thank S. Doyle for assistance in using beamline PDIFF. The Eiger detector at beamline P08 has been funded by the BMBF Project No. 05K10FK2 from the group of Professor Magnussen, University of Kiel.

${ }^{1}$ J. G. Lisoni, M. Siegert, C. Lei, W. Biegel, J. Schubert, W. Zander, and C. Buchal, Thin Solid Films 389, 219-226 (2001).

${ }^{2}$ Y. K. V. Reddy, D. Mergel, S. Reuter, V. Buck, and M. Sulkowski, J. Phys. D: Appl. Phys. 39, 1161-1168 (2006).

${ }^{3}$ W.-C. Shih and M.-H. Chiang, J. Mater. Sci.: Mater. Electron. 21, 844-848 (2009).

${ }^{4}$ G. Li, V. Shrotriya, J. Huang, Y. Yao, T. Moriarty, K. Emery, and Y. Yang, Nat. Mater. 4, 864-868 (2005).

${ }^{5}$ H. A. Atwater and A. Polman, Nat. Mater. 9, 205-213 (2010).

${ }^{6}$ J. van den Hurk, A.-C. Dippel, D. Cho, J. Straquadine, U. Breuer, P. Walter, R. Waser, and I. Valov, Phys. Chem. Chem. Phys. 16, 18217 (2014).

${ }^{7}$ P. Li, T.-M. Lu, and H. Bakhru, Appl. Phys. Lett. 58, 2639 (1991).

${ }^{8}$ G. E. Moore, Electronics 86, 82 (1998).

${ }^{9}$ T. Schneller, R. Waser, M. Kosec, and D. Payne, Chemical Solution Deposition of Functional Oxide Thin Films (Springer, Berlin, 2013).

${ }^{10}$ A.-C. Dippel, T. Schneller, R. Waser, D. Park, and J. Mayer, Chem. Mater. 22, 6209-6211 (2010).

${ }^{11}$ L. Holland, Vacuum Deposition of Thin Films (Wiley, New York, 1956).

${ }^{12}$ M. Ohring, Materials Science of Thin Films (Wiley, New York, 1991).

${ }^{13}$ G. Franz, Low Pressure Plasmas and Microstructuring Technology (Springer-Verlag, Berlin Heidelberg, 2009), chap. 8, pp. 269-294.

${ }^{14} \mathrm{G}$. Franz, Low Pressure Plasmas and Microstructuring Technology (Springer-Verlag, Berlin Heidelberg, 2009), chap. 4, pp. 69-102.

${ }^{15}$ J. A. Thornton, J. Vac. Sci. Technol. 11, 666 (1974).

${ }^{16}$ J. A. Thornton, Annu. Rev. Mater. Sci. 7, 239-260 (1977).

${ }^{17}$ A. Anders, Thin Solid Films 518, 4087-4090 (2010).

${ }^{18}$ J. A. Thornton, J. Vac. Sci. Technol., A 4, 3059 (1986).

${ }^{19}$ S. Förster, M. Huth, K.-M. Schindler, and W. Widdra, J. Chem. Phys. 135, 104701 (2011).

${ }^{20} \mathrm{~J}$. Wang, "Low energy electron diffraction studies of transition metal oxide surfaces and films," Dissertation (Martin-Luther-Universität Halle-Wittenberg, 2005) http://sundoc.bibliothek.uni-halle.de/diss-online/05/05H034/of index.htm.

${ }^{21}$ S. V. Kalinin and A. Gruverman, Scanning Probe Microscopy: Electrical and Electromechanical Phenomena at the Nanoscale (Springer, New York, 2007).

${ }^{22}$ H. Franz, O. Leupold, R. Roehlsberger, S. Roth, O. Seeck, J. Spengler, J. Strempfer, M. Tischer, J. Viefhaus, E. Weckert, and T. Wroblewski, Synchrotron Radiat. News 19, 25-29 (2006).

${ }^{23}$ R. Döhrmann, S. Botta, A. Buffet, G. Santoro, K. Schlage, M. Schwartzkopf, S. Bommel, J. F. H. Risch, R. Mannweiler, S. Brunner, E. Metwalli, P. Müller-Buschbaum, and S. V. Roth, Rev. Sci. Instrum. 84, 043901 (2013).

${ }^{24}$ S. Couet, T. Diederich, K. Schlage, and R. Röhlsberger, Rev. Sci. Instrum. 79, 093908 (2008).

${ }^{25}$ Y. C. Liang, H. Y. Lee, H. J. Liu, Y. W. Hsieh, and Y. C. Liang, J. Synchrotron Radiat. 14, 163-168 (2007). 
${ }^{26}$ D. Carbone, O. Plantevin, R. Gago, C. Mocuta, O. Bikondoa, A. Alija, L. Petit, H. Djazuli, and T. H. Metzger, J. Synchrotron Radiat. 15, 414-419 (2008).

${ }^{27}$ O. Seeck, C. Deiter, K. Pflaum, F. Bertam, A. Beerlink, H. Franz, J. Horbach, H. Schulte-Schrepping, B. M. Murphy, M. Greve, and O. Magnussen, J. Synchrotron Radiat. 19, 30-38 (2011).

${ }^{28}$ S. Venkataraj, O. Kappertz, H. Weis, R. Drese, R. Jayavel, and M. Wuttig, J. Appl. Phys. 92, 3599 (2002).

${ }^{29}$ T. Egami and S. Billinge, Underneath the Bragg Peaks: Structural Analysis of Complex Materials (Elsevier, 2012), Vol. 7.

${ }^{30}$ Tectra http://www.tectra.de/Heater-FAQ.pdf "Heater-faq.pdf Online," (2015).

${ }^{31}$ E. Taurel, J. L. Pons, P. Verdier, J. M. Chaize, J. Meyer, F. Poncet, G. Heunen, J. Horowitz, A. Buteau, N. Leclercq, and M. Ounsy, "Tango a Corba Based Control System," Proceedings of ICALEPCS 2003 (Pohang Accelerator Laboratory, 2003), pp. 220-222.

${ }^{32}$ M. Schwartzkopf, A. Buffet, and V. Körstgens, Nanoscale 5, 5053-5062 (2013).

${ }^{33}$ S. Bommel, N. Kleppmann, C. Weber, H. Spranger, P. Schäfer, J. Novak, S. Roth, F. Schreiber, S. Klapp, and S. Kowarik, Nat. Commun. 5, 5388 (2014).

${ }^{34}$ A.-C. Dippel, H.-P. Liermann, J.-T. Delitz, P. Walter, H. Schulte-Schrepping, O. Seeck, and H. Franz, J. Synchrotron Radiat. 22, 675 (2015).

${ }^{35}$ K. Jensen, H. Andersen, and C. Tyrsted, ACS NANO 8, 10704-10714 (2014).

${ }^{36}$ M. Herklotz, F. Scheiba, M. Hinterstein, K. Nikolowski, M. Knapp, A.-C. Dippel, L. Giebeler, J. Eckert, and H. Ehrenberg, J. Appl. Crystallogr. 46, 1117-1127 (2013).

${ }^{37}$ P. R. Willmott, D. Meister, S. J. Leake, M. Lange, a. Bergamaschi, M. Böge, M. Calvi, C. Cancellieri, N. Casati, a. Cervellino, Q. Chen, C. David, U. Flechsig, F. Gozzo, B. Henrich, S. Jäggi-Spielmann, B. Jakob, I. Kalichava, P. Karvinen, J. Krempasky, a. Lüdeke, R. Lüscher, S. Maag, C. Quitmann, M. L. Reinle-Schmitt, T. Schmidt, B. Schmitt, a. Streun, I. Vartiainen, M. Vitins, X. Wang, and R. Wullschleger, J. Synchrotron Radiat. 20, 667-682 (2013).
${ }^{38}$ E. Wainer and A. N. Solomon, http://scripts.iucr.org/cgi-bin/paper? S0365110X51001768 Titanium Alloy Manufacturing Co. Report No. 8, Vol. 3 (1942).

${ }^{39}$ B. M. Wul and I. M. Goldman, Comput. Rend. Acad. Sci. URSS 46, 154-157 (1945).

${ }^{40}$ T. Ogawa, Busseiron Kenkyo 6, 1-27 (1947) (in Japanese).

${ }^{41}$ P. Vousden, Acta Crystallogr. 4, 545-551 (1951).

${ }^{42}$ J. C. Slater, Phys. Rev. 78, 748-761 (1950), http://prola.aps.org/abstract/PR/ v78/i6/p748_1.

${ }^{43}$ A. V. Hippel, Rev. Mod. Phys. 22 (1950), http://rmp.aps.org/abstract/RMP/ v22/i3/p221_1.

${ }^{44}$ J. A. Gonzalo and B. Jimenez, Ferroelectricity: The Fundamentals Collection (Wiley-VCH, Verlag, Weinheim, 2005).

${ }^{45}$ M. E. Lines and A. M. Glass, Principles and Application of Ferroelectrics and Related Materials (Oxford University Press Inc, New York, 2001).

${ }^{46}$ C. P. d. Araujo, J. F. Scott, and G. W. Taylor, Ferroelectric Thin Films: Synthesis and Basic Properties (Gordon and Breach Publishers, Amsterdam, 1996).

${ }^{47}$ S. Valencia, A. Crassous, L. Bocher, V. Garcia, X. Moya, R. O. Cherifi, C. Deranlot, K. Bouzehouane, S. Fusil, A. Zobelli, a. Gloter, N. D. Mathur, A. Gaupp, R. Abrudan, F. Radu, a. Barthélémy, and M. Bibes, Nat. Mater. 10, 753-758 (2011)

${ }^{48}$ D. A. Tenne, A. Bruchhausen, N. D. Lanzillotti-Kimura, A. Fainstein, R. S. Katiyar, A. Cantarero, A. Soukiassian, V. Vaithyanathan, J. H. Haeni, W. Tian, D. G. Schlom, K. J. Choi, D. M. Kim, C. B. Eom, H. P. Sun, X. Q. Pan, Y. L. Li, L. Q. Chen, Q. X. Jia, S. M. Nakhmanson, K. M. Rabe, and X. X. Xi, Science (New York, N.Y.) 313, 1614-1616 (2006).

${ }^{49}$ V. Garcia, M. Bibes, L. Bocher, S. Valencia, F. Kronast, A. Crassous, X. Moya, S. Enouz-Vedrenne, A. Gloter, D. Imhoff, C. Deranlot, N. D. Mathur, S. Fusil, K. Bouzehouane, and a. Barthélémy, Science 327, 1106-1110 (2010).

${ }^{50}$ D. Y. Noh, H. H. Lee, T. S. Kang, and J. H. Je, Appl. Phys. Lett. 72, 2823 (1998).

${ }^{51}$ L. G. Parratt, Phys. Rev. 95, 359-369 (1954). 